\title{
Number of degrees of freedom and energy spectrum of surface quasi-geostrophic turbulence
}

\author{
Chuong V. Tran $\dagger$, Luke A. K. Blackbourn and Richard K. Scott \\ School of Mathematics and Statistics, University of St Andrews, St Andrews KY16 9SS, UK
}

(Received 22 July 2010; revised 22 April 2011; accepted 14 July 2011; first published online 5 September 2011)

We study both theoretically and numerically surface quasi-geostrophic turbulence regularized by the usual molecular viscosity, with an emphasis on a number of classical predictions. It is found that the system's number of degrees of freedom $N$, which is defined in terms of local Lyapunov exponents, scales as $R e^{3 / 2}$, where $R e$ is the Reynolds number expressible in terms of the viscosity, energy dissipation rate and system's integral scale. For general power-law energy spectra $k^{-\alpha}$, a comparison of $N$ with the number of dynamically active Fourier modes, i.e. the modes within the energy inertial range, yields $\alpha=5 / 3$. This comparison further renders the scaling $R e^{1 / 2}$ for the exponential dissipation rate at the dissipation wavenumber. These results have been predicted on the basis of Kolmogorov's theory. Our approach thus recovers these classical predictions and is an analytic alternative to the traditional phenomenological method. The implications of the present findings are discussed in conjunction with related results in the literature. Support for the analytic results is provided through a series of direct numerical simulations.

Key words: geostrophic turbulence, quasi-geostrophic flows

\section{Introduction}

A popular model of geophysical fluids arising from the theory of quasi-geostrophy is governed by the advection-diffusion equation (Pedlosky 1987)

$$
\theta_{t}+\mathbf{J}(\psi, \theta)=v \Delta \theta,
$$

which is known as the surface quasi-geostrophic (SQG) equation. In (1.1), $\theta(x, y, t)$ is the surface potential temperature of a three-dimensional quasi-geostrophic fluid, in which the interior potential vorticity is assumed to be identically zero, $\psi(x, y, t)$ is the streamfunction, $\mathrm{J}(\cdot, \cdot)$ denotes the Jacobian and $v$ is the viscosity. The potential temperature is related to the streamfunction by $\theta(x, y, t)=(-\Delta)^{1 / 2} \psi(x, y, t)$, where $\Delta$ is the Laplacian. We use the usual molecular viscosity in order to treat SQG turbulence on an equal footing with three-dimensional Navier-Stokes turbulence and one-dimensional Burgers flows. Equation (1.1) is important for its relevance to surface dynamics in the atmospheres and oceans, including the dynamics of the tropopause (e.g. Juckes 1994). In addition, close analogies between SQG and three-dimensional

$†$ Email address for correspondence: chuong@mcs.st-and.ac.uk 
Navier-Stokes turbulence concerning the issue of solution regularity have made (1.1) a subject of mathematical interest (cf. Constantin, Majda \& Tabak 1994; Kiselev, Nazarov \& Volberg 2007; Ohkitani 2011).

The relation between $\psi$ and $\theta$ may be more conveniently represented in Fourier space, taking, for simplicity, a periodic domain of size $L \times L$, and assuming all fields to have zero spatial average. For real $\gamma$, the differential operator $(-\Delta)^{\gamma / 2}$ is defined by $\left(-\widehat{\Delta)^{\gamma / 2}} \psi\left(k_{1}, k_{2}, t\right)=k^{\gamma} \widehat{\psi}\left(k_{1}, k_{2}, t\right)\right.$, where $k=\left(k_{1}^{2}+k_{2}^{2}\right)^{1 / 2}$ is the wavenumber and $\left(-\widehat{\Delta)^{\gamma / 2}} \psi\left(k_{1}, k_{2}, t\right)\right.$ and $\widehat{\psi}\left(k_{1}, k_{2}, t\right)$ are the Fourier transforms of $(-\Delta)^{\gamma / 2} \psi(x, y, t)$ and $\psi(x, y, t)$, respectively. It follows that the Fourier transform $\widehat{\theta}\left(k_{1}, k_{2}, t\right)$ of $\theta(x, y, t)$ is expressible in terms of $\widehat{\psi}\left(k_{1}, k_{2}, t\right)$ by

$$
\widehat{\theta}\left(k_{1}, k_{2}, t\right)=k \widehat{\psi}\left(k_{1}, k_{2}, t\right) .
$$

When $v=0,(1.1)$ describes the material conservation of the potential temperature $\theta$ (on an impermeable horizontal surface). This gives rise to the conservation of the temperature variance, $\|\theta\|^{2}=\left\langle\theta^{2}\right\rangle$, where $\langle\cdot\rangle$ denotes a spatial average. By virtue of (1.2) one has $\|\theta\|=\|\nabla \psi\|$, so that the fluid kinetic energy $\|\boldsymbol{u}\|^{2} / 2=\|\nabla \psi\|^{2} / 2$ is conserved. Another conserved quantity of SQG turbulence is $\langle\psi \theta\rangle=\left\|(-\Delta)^{1 / 4} \psi\right\|^{2}=$ $\left\|(-\Delta)^{-1 / 4} \theta\right\|^{2}$. In general, $\left\|(-\Delta)^{-1 / 4} \theta\right\|^{2}$ and $\|\theta\|^{2}$ are preferentially transferred to large and small scales, respectively. This dual transfer imposes well-known constraints on each individual transfer. For example, within a given wavenumber triad, energy is transferred from the intermediate wavenumber to both the lower and higher wavenumbers (or vice versa). This is in a sharp contrast to Burgers flows, for which energy can be transferred from the lower and intermediate wavenumbers to the higher one (Tran \& Dritschel 2010).

Equation (1.1) with or without a dissipation term has been a subject of active mathematical, phenomenological and numerical research. Mathematical studies, initiated by Constantin et al. (1994), have been mainly concerned with the issue of solution regularity. The reason for this particular interest in the SQG equation is that, by virtue of (1.2), the power spectra of the advecting flow $\boldsymbol{u}=\left(-\psi_{y}, \psi_{x}\right)$ and of the active scalar $\theta$ are identical. Hence $\theta$ is a same-scale copy of $\boldsymbol{u}$. This means that the advection terms in SQG and three-dimensional turbulence have the same 'effective degree of nonlinearity' (see Tran, Dritschel \& Scott 2010). It was hoped that an understanding of the regularity problem in the SQG equation would shed light on the same problem in the Navier-Stokes system. It has been found that dissipation mechanisms of the form $(-\Delta)^{\gamma / 2} \theta$, where $1 \leqslant \gamma \leqslant 2$, are sufficiently strong to regularize the SQG dynamics (see Kiselev et al. 2007 and references therein). Hence, (1.1) possesses global smooth solutions. Phenomenological studies, initiated by Pierrehumbert, Held \& Swanson (1994), have applied Kolmogorov's theory to SQG turbulence to predict the spectral scaling $k^{-5 / 3}$ for the energy inertial range. Thus far, there has been no other theoretical evidence for this spectrum, while numerical evidence has been limited and inconclusive (see, for example, Held et al. 1995; Ohkitani \& Yamada 1997; Smith et al. 2002; Scott 2006; Capet et al. 2008).

In this study, we make use of the dynamical systems approach of Tran \& Blackbourn (2009) (see also Tran 2009; Tran \& Dritschel 2010) to derive a number of classical results, which have been predicted on the basis of Kolmogorov's phenomenology. These include an estimate for the number of degrees of freedom of SQG turbulence and its immediate consequences. This number, denoted by $N$, is found to scale as $R e^{3 / 2}$, where $R e$ is the Reynolds number defined in terms of the 
system's length scale, the energy dissipation rate and the viscosity. By comparing $N$ with the number of dynamically active Fourier modes within the inertial range having a general energy spectrum $k^{-\alpha}$, we recover the classical result $\alpha=5 / 3$. This comparison further renders the scaling $R e^{1 / 2}$ for the exponential dissipation rate at the dissipation wavenumber. The present findings are briefly discussed in conjunction with previous results in the literature for one-dimensional Burgers flows and two- and three-dimensional turbulence.

Finally, evidence for the $k^{-5 / 3}$ spectrum is provided through a series of direct numerical simulations of (1.1), in which the energy is initially located at intermediate scales sufficiently far away from the domain scale as to ensure unnoticeable contamination of the inverse transfer up to the required time. The numerical results confirm the scaling $N \approx R e^{3 / 2}$ and support the classical hypothesis of finite energy dissipation in the inviscid limit. Using an initial condition with a relatively high degree of isotropy, the maximum energy dissipation rate decreases with increasing $R e$ for moderate values of $R e$, nonetheless appearing to become independent of $R e$ for large $R e$. On the other hand, when the initial flow is relatively more anisotropic, allowing for certain classes of Fourier modes to be subsequently excited, the maximum energy dissipation rate is more sharply peaked in time, presumably growing towards a finite value as $R e$ is increased.

\section{Lyapunov stability analysis and number of degrees of freedom}

\subsection{Preliminaries}

We recall two analytic inequalities and an identity used in this study. Consider the set of doubly periodic and zero-average functions $\left\{\vartheta_{1}, \vartheta_{2}, \ldots, \vartheta_{n}\right\}$ satisfying the orthonormality conditions $\left\langle\vartheta_{i} \vartheta_{j}\right\rangle=\delta_{i j}$, where $\delta_{i j}$ is the Kronecker delta symbol. For the present case of a doubly periodic domain of size $L$, the eigenvalues under $-\Delta$ of the first $n$ basic Fourier modes sum up to approximately $n^{2} / L^{2}$. The Rayleigh-Ritz principle implies (cf. Tran \& Blackbourn 2009)

$$
\sum_{i=1}^{n}\left\|\nabla \vartheta_{i}\right\|^{2} \geqslant c_{1} \frac{n^{2}}{L^{2}},
$$

where $c_{1}$ is a non-dimensional constant independent of the given set and of its size $n$. On the other hand, we have the Lieb-Thirring inequality (cf. Constantin, Foias \& Temam 1988; Temam 1997; Tran \& Blackbourn 2009)

$$
\left\|\sum_{i=1}^{n} \vartheta_{i}^{2}\right\| \leqslant c_{2} L\left(\sum_{i=1}^{n}\left\|\nabla \vartheta_{i}\right\|^{2}\right)^{1 / 2},
$$

where $c_{2}$ is a non-dimensional constant, which is, like $c_{1}$, independent of the given set and of its size $n$.

For doubly periodic functions $\vartheta, \varphi$ and $\phi$ whose gradients have finite norms, we have, by integration by parts,

$$
\langle\vartheta \mathrm{J}(\varphi, \phi)\rangle=-\langle\varphi \mathrm{J}(\vartheta, \phi)\rangle=-\langle\phi \mathrm{J}(\varphi, \vartheta)\rangle .
$$

This gives rise to the well-known conservation laws discussed in the introductory section. 
The decay of energy is governed by

$$
\frac{1}{2} \frac{\mathrm{d}}{\mathrm{d} t}\|\theta\|^{2}=-v\|\nabla \theta\|^{2}=-\epsilon(t) .
$$

Given a smooth initial temperature field, say $\theta_{0}(x, y)=\theta(x, y, 0)$, and fixed $v>0$, the dissipation rate $\epsilon(t)$ is bounded (because of global smoothness of solutions) and eventually decays to zero. As we are interested in high Reynolds numbers, the relevant dynamical behaviour is that $\|\nabla \theta\|$ grows initially. The dissipation rate then achieves a global maximum, say $\epsilon_{T}=\epsilon(T)$, at some time $t=T$. In general, $\epsilon_{T}$ and $T$ may depend on $v$ - a possibility intimately related to the question of finite-time blowup of inviscid solutions. For a quantitative sense of this issue, we note that, in the inviscid limit, both $\epsilon_{T}$ and $T$ are independent of $v$ for shock-dominated Burgers flows (cf. Tran \& Dritschel 2010). Such properties constitute the key hypothesis of Kolmogorov's theory of three-dimensional turbulence. On the other hand, for two-dimensional turbulence in the same limit, the maximum enstrophy dissipation rate (the parallel of $\epsilon_{T}$ ) vanishes and $T$ diverges (Tran \& Dritschel 2006; Dritschel, Tran \& Scott 2007). For the present case, Ohkitani \& Yamada (1997) suggested similar behaviours for $\epsilon_{T}$ and $T$, thus favouring the possibility of no finite-time singularities in the inviscid dynamics. In a study using the very initial condition of Ohkitani \& Yamada (1997), Constantin, Nie \& Schörghofer (1998) suggested no finite-time blowup. If this is indeed the case, then $\epsilon(t) \rightarrow 0$ as $v \rightarrow 0$ for all $t<\infty$ because $\|\nabla \theta\|<\infty$. However, the possibility that $\|\nabla \theta\|$ diverges as $t \rightarrow \infty$ cannot be ruled out. This singularity at infinity is in fact probable. This means that further investigation is needed to determine whether or not $\epsilon_{T} \rightarrow 0$. In any case, the detailed behaviour of $\epsilon_{T}$ is not a major concern in the present study (more detail in due course). In what follows, we make use of $\epsilon_{T}$ in key derivations, pending further knowledge of this important dynamical quantity.

\subsection{Lyapunov stability analysis}

For a regular forced-dissipative infinite-dimensional dynamical system, solutions starting from finite initial conditions can asymptotically approach a universal set in phase space (Fourier space). Such a set is known as a global attractor, which is invariant and represents the long-term dynamical behaviour of the system in question. In general, an attractor is fractal and has a finite Hausdorff (or box-counting) dimension, say $D_{H}$. This dimension has been considered the system's number of degrees of freedom, in the sense that the attractor is essentially a $D_{H}$-dimensional object, to which all solutions converge. A number of authors have estimated $D_{H}$ for fluid systems such as the two-dimensional Navier-Stokes equations (see, for example, Constantin et al. 1988; Doering \& Gibbon 1991; Robinson 2003). For unforceddissipative systems, the concept of an attractor is irrelevant since the asymptotic dynamics are trivial. Nevertheless, the notion of number of degrees of freedom remains meaningful for the transient dynamics. For a given solution at a given time, Tran \& Blackbourn (2009) identified this number with the dimension of the subspace spanned by a sufficiently large number of least Lyapunov stable modes of the system linearized about the solution in question. Here, 'sufficiently large' means that the sum of Lyapunov exponents of these modes becomes negative. For the present case, the problem of Lyapunov stability and number of degrees of freedom is formulated and further explained in what follows (cf. Tran 2009; Tran \& Blackbourn 2009; Tran \& Dritschel 2010).

Given the solution $\theta(x, y, t)$ (with the corresponding streamfunction $\psi$ ) starting from some smooth initial temperature field $\theta_{0}(x, y)$, consider a disturbance $\vartheta$ to $\theta$ 
(corresponding to a disturbance $\varphi$ to $\psi$ ). The linear evolution of $\vartheta$ is governed by

$$
\vartheta_{t}+\mathbf{J}(\varphi, \theta)+\mathbf{J}(\psi, \vartheta)=v \Delta \vartheta
$$

By multiplying (2.5) by $\vartheta$ and taking the spatial average of the resulting equation, we obtain the evolution equation for the disturbance norm $\|\vartheta\|$ :

$$
\|\vartheta\| \frac{\mathrm{d}}{\mathrm{d} t}\|\vartheta\|=-\langle\vartheta \mathrm{J}(\varphi, \theta)\rangle-v\|\nabla \vartheta\|^{2},
$$

where one of the triple-product terms, namely $\langle\vartheta \mathrm{J}(\psi, \vartheta)\rangle$, vanishes due to (2.3). It follows that the exponential growth or decay rate $\lambda$ of $\|\vartheta\|$ is given by

$$
\lambda=\frac{1}{\|\vartheta\|} \frac{\mathrm{d}}{\mathrm{d} t}\|\vartheta\|=-\frac{1}{\|\vartheta\|^{2}}\left(\langle\vartheta \mathrm{J}(\varphi, \theta)\rangle+v\|\nabla \vartheta\|^{2}\right) .
$$

An orthonormal set of $n$ least stable disturbances $\left\{\vartheta_{1}, \vartheta_{2}, \ldots, \vartheta_{n}\right\}$ (with the associated set of streamfunctions $\left\{\varphi_{1}, \varphi_{2}, \ldots, \varphi_{n}\right\}$, where $\left.(-\Delta)^{1 / 2} \varphi_{i}=\vartheta_{i}\right)$ and the corresponding greatest growth rates $\left\{\lambda_{1}, \lambda_{2}, \ldots, \lambda_{n}\right\}$ (local Lyapunov exponents) can be derived by successively maximizing $\lambda$ with respect to all admissible disturbances $\vartheta$ subject to the following orthogonality constraint. At each step $i$ in the process, the disturbance $\vartheta$ is required to satisfy both $\|\vartheta\|=1$ and $\left\langle\vartheta \vartheta_{j}\right\rangle=0$, for $j=1,2, \ldots, i-1$, where $\vartheta_{j}$ is the maximizer obtained at the $j$ th step. This process eventually exhausts all unstable mutually orthogonal disturbances and reaches the stable regime where $\lambda_{i}<0$. It follows that there exists an integer $N$ satisfying

$$
\sum_{i=1}^{N} \lambda_{i} \leqslant 0<\sum_{i=1}^{N-1} \lambda_{i} .
$$

The orthonormal set $\left\{\vartheta_{1}, \vartheta_{2}, \ldots, \vartheta_{N}\right\}$ then consists of all unstable modes and a number of stable modes, which can adequately describe the solution $\theta$ at least locally in time. In other words, the $N$-dimensional linear subspace spanned by $\left\{\vartheta_{1}, \vartheta_{2}, \ldots, \vartheta_{N}\right\}$ adequately 'accommodates' the solution. Indeed, for theoretical purposes, one can formally express $\theta$ in terms of this spanning set (instead of the Fourier modes), with the understanding that the 'truncation' mode $\vartheta_{N}$ lies well within the dissipation range (large negative Lyapunov exponent). For this reason, $N$ has been called the number of degrees of freedom (see Tran \& Blackbourn 2009; Tran 2009; Tran \& Dritschel 2010). As is clear from the formulation, $N$ is both solution- and time-dependent. In general, the spanning set $\left\{\vartheta_{1}, \vartheta_{2}, \ldots, \vartheta_{N}\right\}$ may change from time to time and from one solution to another. This study (as well as the cited papers), however, considers a general solution at an arbitrary time and estimates $N$ under such general conditions. Therefore, the applicability of the present notion of number of degrees of freedom is quite broad. Thus, the derived estimate for $N$ can be legitimately called the system's number of degrees of freedom in a general sense.

\subsection{An estimate for the number of degrees of freedom}

Since each normalized solution $\left(\lambda_{i}, \vartheta_{i}\right)$ satisfies (2.7), we have

$$
\begin{aligned}
\sum_{i=1}^{n} \lambda_{i} & =-\sum_{i=1}^{n}\left(\left\langle\vartheta_{i} \mathrm{~J}\left(\varphi_{i}, \theta\right)\right\rangle+v\left\|\nabla \vartheta_{i}\right\|^{2}\right) \\
& \leqslant \sum_{i=1}^{n}\left(\left\langle\left|\vartheta_{i}\right|\left|\nabla \varphi_{i}\right||\nabla \theta|\right\rangle-v\left\|\nabla \vartheta_{i}\right\|^{2}\right)
\end{aligned}
$$




$$
\begin{aligned}
& \leqslant\|\nabla \theta\|\left\|\sum_{i=1}^{n} \vartheta_{i}^{2}\right\|^{1 / 2}\left\|\sum_{i=1}^{n}\left|\nabla \varphi_{i}\right|^{2}\right\|^{1 / 2}-v \sum_{i=1}^{n}\left\|\nabla \vartheta_{i}\right\|^{2} \\
& \leqslant c_{2} L\|\nabla \theta\|\left(\sum_{i=1}^{n}\left\|\nabla \vartheta_{i}\right\|^{2}\right)^{1 / 2}-v \sum_{i=1}^{n}\left\|\nabla \vartheta_{i}\right\|^{2} \\
& \leqslant\left(\sum_{i=1}^{n}\left\|\nabla \vartheta_{i}\right\|^{2}\right)^{1 / 2}\left(c_{2} L \frac{\epsilon_{T}^{1 / 2}}{v^{1 / 2}}-c_{1}^{1 / 2} v \frac{n}{L}\right) .
\end{aligned}
$$

In (2.9), we have used the Cauchy-Schwarz inequality in the third step, the Lieb-Thirring inequality (2.2), together with the slightly different but equivalent version

$$
\left\|\sum_{i=1}^{n}\left|\nabla \varphi_{i}\right|^{2}\right\| \leqslant c_{2} L\left(\sum_{i=1}^{n}\left\|\Delta \varphi_{i}\right\|^{2}\right)^{1 / 2}=c_{2} L\left(\sum_{i=1}^{n}\left\|\nabla \vartheta_{i}\right\|^{2}\right)^{1 / 2},
$$

in the penultimate step, and (2.1) in the final step. Note that, in (2.10), we have used the identity $\left\|\Delta \varphi_{i}\right\|=\left\|\nabla \vartheta_{i}\right\|$. The condition $\sum_{i=1}^{n} \lambda_{i} \leqslant 0$ is satisfied when $n \geqslant c_{2} L^{2} \epsilon_{T}^{1 / 2} /\left(c_{1}^{1 / 2} v^{3 / 2}\right)$. It follows that

$$
N \leqslant \frac{c_{2}}{c_{1}^{1 / 2}} \frac{L^{2} \epsilon_{T}^{1 / 2}}{v^{3 / 2}}=\frac{c_{2}}{c_{1}^{1 / 2}}\left(\frac{L^{4 / 3} \epsilon_{T}^{1 / 3}}{v}\right)^{3 / 2}=R e^{3 / 2},
$$

where $R e$ is the Reynolds number, defined in the same way as in three-dimensional Navier-Stokes turbulence (Tran 2009).

The estimate (2.11) can be optimal up to a factor not tending to zero as rapidly as $R e^{-\gamma}$, for $\gamma>0$. In other words, the exponent $3 / 2$ of $\operatorname{Re}^{3 / 2}$ can be exact. The reason is that all the inequalities used in (2.9) are relatively sharp. For example, by virtue of (2.1) and (2.2)), the norm $\left\|\sum_{i=1}^{n} \vartheta_{i}^{2}\right\|$ amounts to approximately $n$. This is an optimal estimate as there are no reasons to expect this norm to be of order $n^{\gamma}$ for $\gamma<1$. The sharpness of this most sophisticated estimate in (2.9) provides us with confidence in the optimality of (2.11). In three-dimensional turbulence, the situation is not so optimistic. For this case, a similar line of estimates for the number of degrees of freedom yields the bound $N \leqslant R e^{18 / 5}$ (see the discussion of Tran 2009 and references therein). When compared with the improved estimate $N \approx R e^{9 / 4}$, which is the Landau prediction on the basis of Kolmogorov's theory, this bound is an overestimate by a factor of $R e^{27 / 20}$. Tran (2009) attributes this to the excessive nature of the Lieb-Thirring inequality in three dimensions (when applied to turbulence). In order to appreciate this claim, we note that the norm $\left\|\sum_{i=1}^{n} \boldsymbol{v}_{i}^{2}\right\|$, where $\boldsymbol{v}_{i}$ is the three-dimensional counterpart of $\vartheta_{i}$, when estimated by (7) and (25) of Tran (2009) - the three-dimensional counterparts of (2.1) and (2.2) - amounts to $n^{5 / 4}$. There is an apparent difference by a factor of $n^{1 / 4}$ for the values of $\left\|\sum_{i=1}^{n} \vartheta_{i}^{2}\right\|$ and $\left\|\sum_{i=1}^{n} \boldsymbol{v}_{i}^{2}\right\|$. This implies that the Lieb-Thirring inequality can be potentially excessive by a factor of $n^{1 / 4}$ in the context of three-dimensional Navier-Stokes turbulence. Interestingly, if this factor were to be removed, one would then recover the Landau prediction. In $\$ 4$ the present result $N \leqslant R e^{3 / 2}$ is fully justified by our numerical simulations of (1.1), where adequate dissipation is observed when we set the ratio $N / \operatorname{Re}^{3 / 2}$ (more precisely the product $v^{3 / 2} k_{\max }^{2}$, where $k_{\max } \approx N^{1 / 2} / L$ is the truncation wavenumber) at order unity. 
This can be considered numerical evidence for the result (2.11) and is discussed in more detail in the subsequent section.

\section{Inertial range scaling}

This section makes use of the analytic estimate (2.11) to deduce the spectral scaling $k^{-5 / 3}$ for the energy inertial range. This approach can be seen as an analytic alternative to the familiar phenomenological dimensional analysis method. We briefly discuss the implication of the present results in conjunction with related results in the literature.

\subsection{The classical spectrum $k^{-5 / 3}$}

At the instant of maximum energy dissipation, consider the power-law spectrum $C k^{-\alpha}$ of the inertial range extending to some dissipation wavenumber $k_{v}$, where $C$ is a constant of suitable dimension. For such a spectrum, it is straightforward to deduce $\alpha$ from (2.11) under the assumption that the number of Fourier modes within the inertial range, say $N^{\prime}$, is comparable to $N$. To this end, we note that $\epsilon_{T} \approx \nu C k_{v}^{3-\alpha} /(3-\alpha)$, assuming $\alpha<3$. It follows that $N^{\prime}$ is given by

$$
N^{\prime} \approx L^{2} k_{v}^{2} \approx L^{2}\left(\frac{3-\alpha}{C v} \epsilon_{T}\right)^{2 /(3-\alpha)}
$$

From our assumption $N \approx N^{\prime}$ and the two estimates (2.11) and (3.1) we deduce that

$$
\left(\frac{\epsilon_{T}}{C \nu}\right)^{2 /(3-\alpha)} \approx \frac{\epsilon_{T}^{1 / 2}}{\nu^{3 / 2}},
$$

where the respective constant factors $(3-\alpha)^{2 /(3-\alpha)}$ and $c_{2} / c_{1}^{1 / 2}$ on the left-hand and right-hand sides have been omitted. It follows that

$$
\epsilon_{T}^{1+\alpha} \approx C^{4} v^{3 \alpha-5}
$$

Given $\epsilon_{T}>0$ in the inviscid limit, (3.3) implies $\alpha=5 / 3$. Thus we have recovered the classical spectrum by the present method. Furthermore, for this value of $\alpha$, the constant $C$ can be seen to be proportional to $\epsilon_{T}^{2 / 3}$, consistent with the Kolmogorov spectrum $C_{K} \epsilon_{T}^{2 / 3} k^{-5 / 3}$, where $C_{K}$ is the Kolmogorov constant. In passing, it is worth noting that the a priori condition $\epsilon_{T}>0$ is not crucial and can be relaxed to a certain extent. In particular, the result $\alpha=5 / 3$ remains valid when $\epsilon_{T}$ tends to zero logarithmically (see the concluding remarks in $\S 5$ for more detail).

\subsection{Discussion}

It is remarkable that no values of $\alpha$ other than $5 / 3$ are admissible, provided that the estimate (2.11) is optimal in the sense discussed in the preceding section. This result can be 'half' anticipated for the following reason. Onsager (1949) conjectured that solutions of the three-dimensional Euler equations satisfying $|\boldsymbol{u}(\boldsymbol{x}+\boldsymbol{r})-\boldsymbol{u}(\boldsymbol{x})| \propto|\boldsymbol{r}|^{\gamma}$, where $\gamma>1 / 3$, conserve energy. Such solutions correspond to energy spectra steeper than $k^{-5 / 3}$ and indeed have been shown to conserve energy (Constantin, E \& Titi 1994). Equation (3.3) is consistent with this result since $\alpha>5 / 3$ readily implies that $\epsilon_{T}$ vanishes in the inviscid limit. On the other hand, the regime $\alpha<5 / 3$ would require $\epsilon_{T}$ to grow without bound in the same limit, again provided that (2.11) is optimal. Such a behaviour would be an insult to the Kolmogorov spirit and is highly improbable for smooth solutions satisfying the decay (2.4). 
We have earlier equated the number of active Fourier modes $N^{\prime}$ within the energy inertial range with the derived number of degrees of freedom $N$. This amounts to $k_{v}^{2} \approx N / L^{2} \approx \epsilon_{T}^{1 / 2} / \nu^{3 / 2}$. It follows that the exponential dissipation rate $r=v k_{v}^{2}$ at the dissipation wavenumber $k_{v}$ is given by

$$
r \approx \frac{\epsilon_{T}^{1 / 2}}{v^{1 / 2}} .
$$

Hence $r$ grows as $R e^{1 / 2}$ when $R e$ is increased. By setting $k_{\max }=k_{v}$ in numerical simulations, we have the practical version of (3.4)

$$
v \approx \frac{\epsilon_{T}^{1 / 3}}{k_{\max }^{4 / 3}},
$$

which is used in $\S 4$ for setting $v$ in numerical simulations at various resolutions. Note that (3.4) is a straightforward consequence of the classical spectrum $C_{K} \epsilon_{T}^{2 / 3} k^{-5 / 3}$. Here it has been derived independently of this scaling, just assuming $N^{\prime} \approx N$. For a comparison, this same rate scales as $R e^{1}, R e^{1 / 2}$ and $R e^{0}$ for one-dimensional Burgers flows, three- and two-dimensional turbulence, respectively (Tran \& Dritschel 2010). The difference in $r$ for these systems can be attributed to fundamental differences in their dynamics and is worthy of a closer look. The scaling $r \propto R e^{0}$ in two-dimensional turbulence has long been expected and is well known to numerical analysts. This is due to the effectively linear behaviour of the vorticity gradient dynamics (Tran et al. 2010), rendering a relatively weak excitation of the small scales. This excitation is balanced by viscous effects at the scale where $r \propto R e^{0}$. The dependence $r \propto R e$ in Burgers flows reflects the fully quadratic nonlinearities of the velocity gradient dynamics, resulting in a relatively intense excitation of the small scales. This excitation is the strongest of all these cases, reaching the scale with $r \propto R e$, whereby viscous forces become sufficiently strong to balance nonlinear effects. Now, the 'intermediate' scaling $r \propto R e^{1 / 2}$ in both SQG and three-dimensional turbulence means that relatively mild dissipation at $k_{v}$ is needed for the same purpose. This implies some partial depletion of nonlinear effects (from fully quadratic nonlinearities), which has been widely discussed and studied from different perspectives (Hou \& Li 2006, 2008). This topic is beyond the scope of the present study and is briefly mentioned here in passing. A manifestation of this depletion is the relatively shallow $k^{-5 / 3}$ spectrum compared with its $k^{-2}$ shock-dominated counterpart. This is consistent with the intuitive expectation that weaker nonlinearities allow for shallower spectra to develop - a justifiable fact on the basis of the present analysis. Indeed, weaker nonlinearities correspond to better Lyapunov stability, thereby implying smaller $N$ and $r$. This in turn implies smaller $\alpha$, i.e. shallower spectra.

Numerical evidence for the spectrum $k^{-5 / 3}$ in the literature is rather limited and inconclusive. For example, by directly simulating (1.1) without any modifications, Ohkitani \& Yamada (1997) found an energy spectrum close to $k^{-2}$. A similar attempt by Capet et al. (2008), but with the usual molecular viscosity term replaced by a hyperviscosity term, found a slightly shallower spectrum: between $k^{-2}$ and $k^{-5 / 3}$. Intuitively, these steep spectra may be attributable to low numerical resolutions, i.e. low Reynolds numbers. Another reason for the observed steep spectrum in Ohkitani \& Yamada (1997) is due to the choice of the initial condition $\theta_{0}=\sin x \sin y+\cos y$, which consists of the lowest $(k=1)$ and second lowest $(k=\sqrt{2})$ wavenumbers only. For this initial condition, $\left\|\theta_{0}\right\|^{2}=3 / 4$ and $\left\|(-\Delta)^{-1 / 4} \theta_{0}\right\|^{2}=1 / 2+1 /(4 \sqrt{2})$. So by the dual conservation of $\|\theta\|^{2}$ and $\left\|(-\Delta)^{-1 / 4} \theta\right\|^{2}$, even in the idealized situation whereby 
all of $\left\|(-\Delta)^{-1 / 4} \theta\right\|^{2}$ got transferred to $k=1$, no more than $9.8 \%[(1-1 / \sqrt{2}) / 3]$ of the energy would become available for transfer to the higher wavenumbers $(k \geqslant 2)$. In practice, much less energy is available for transfer since the wavenumber $k=\sqrt{2}$ may not become completely depleted of energy. This inevitably results in weak smallscale dynamics and a steep spectrum. On the other hand, using both hyperviscosity and large-scale dissipation terms (plus a forcing term), Scott (2006) obtained energy spectra close to $k^{-5 / 3}$. With modern computers, it is quite possible to confirm the present prediction by a series of simulations with fixed $\theta_{0}$ and increasingly higher resolutions (with corresponding increasingly higher Reynolds numbers). Note that in accord with (3.5), doubling the resolution (i.e. doubling $k_{\max }$ or quadrupling $N$ ) allows for the viscosity to be reduced by a factor of $2^{-4 / 3}$. The same reduction factor applies to numerical simulations of three-dimensional turbulence (see the discussion of Tran \& Dritschel 2010).

\section{Numerical results}

We now present the results from numerical simulations that support both the above predictions and the underlying hypothesis that the maximum dissipation rate $\epsilon_{T}$ tends to a non-zero limit in the inviscid limit. Equation (1.1) was integrated numerically using a standard pseudo-spectral method in a doubly periodic square domain of side $2 \pi$ with resolutions up to $4096 \times 4096$ grid points. The time stepping is a fourthorder Runge-Kutta scheme, with the viscous term incorporated exactly through an integrating factor. A number of initial temperature fields were considered, differing in the distribution of the initial energy spectrum. Using an initial field comprising Fourier modes $(5,6),(6,5)$ and $(6,6)$ allows for a direct cascading range of almost two decades in wavenumber at the highest resolution used, as well as a modest inverse cascading range. The latter is wide enough to ensure negligible contamination of the inverse transfer by the spectral boundary at $k=1$. Indeed, by the time of maximum energy dissipation, we observed negligible finite-size effects, with the largest scales remaining only weakly excited.

Support for the scaling (3.5) was obtained by defining the coefficient of viscosity as $v=c k_{\max }^{-4 / 3}$, where $c$ is a numerical constant determined as being just large enough to ensure numerical stability. For values of $k_{\max }$ between 128 and 2048 (i.e. spatial grids between $256^{2}$ and $4096^{2}$ ) a value of $c=10 / 64$ was found in all cases to be sufficient to prevent large spikes in the large-wavenumber tail of the energy spectrum (see figure 3 and associated discussion below).

Figure 1 shows the temperature fields for integrations at grid resolutions 1024, 2048 and 4096 at $t=9.2$, which is approximately the time of maximum energy dissipation in all three cases (see figure 2). Note that, unlike two-dimensional turbulence, for which, by the time of peak enstrophy dissipation, initial vorticity fields are typically distorted beyond recognition, the basic pattern of the initial temperature field can still be recognized in figure 1 . The reason for this difference is rather obvious and has its root in the discrepancy in the effective degrees of nonlinearity between the two systems (Tran et al. 2010). In two-dimensional turbulence, the enstrophy is redistributed more or less uniformly among the wavenumber octaves of the inertial range until it reaches the dissipation scale. This transient and undissipated cascade obliterates the initial fields. In contrast, in the present case, the initial temperature field does not change dramatically before the time $t=T$ because relatively little energy is transferred to the small scales during the early stages of the evolution. 
(a)

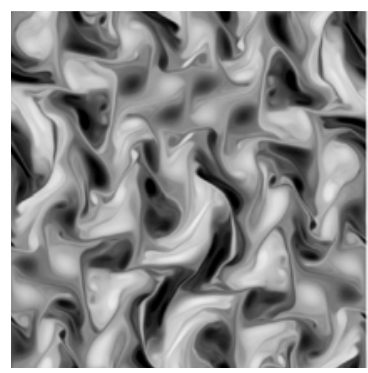

(b)

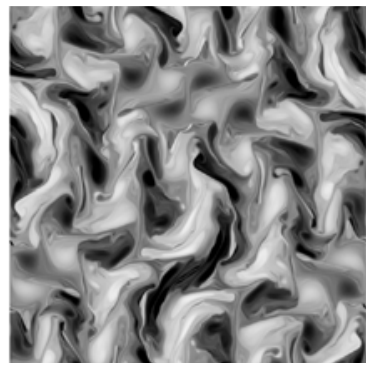

(c)

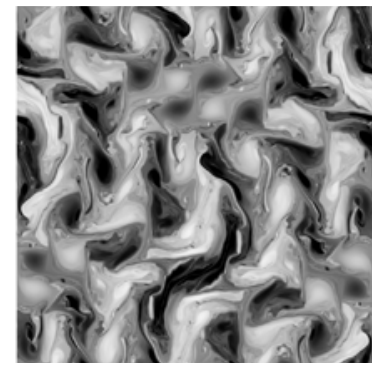

FIGURE 1. The temperature fields $\theta(x, y, T)$ at time of peak energy dissipation from numerical integrations with grid resolutions of (a) 1024, (b) 2048 and (c) 4096.

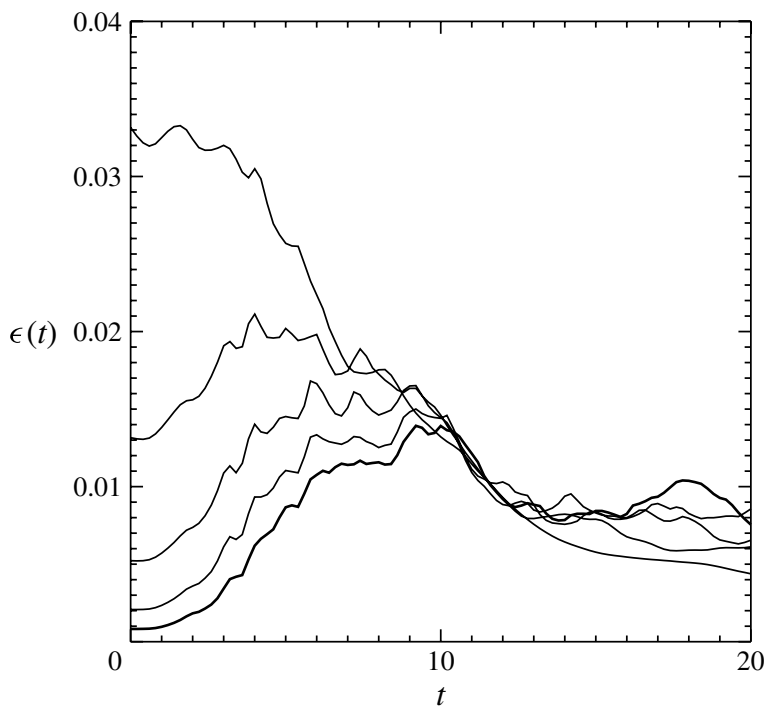

FIgURE 2. The energy dissipation rate $\epsilon(t)$ versus $t$ for numerical integrations with resolutions $256,512,1024,2048,4096$ (bold) and diffusion coefficient $v=(10 / 64) k_{\max }^{-4 / 3}$ (ranging from $v \approx 2.42 \times 10^{-4}$ to $v \approx 6.01 \times 10^{-6}$ ).

Figure 2 shows the evolution of the energy dissipation rate $\epsilon(t)=v\|\nabla \theta\|^{2}$ from $t=0$ to $t=20$. Note that the initial energy in each case is 0.375 . For small $t$, there is significant dissipation in the low-resolution cases resulting from the direct action of the viscosity on the energy-containing scales of the initial temperature field. As resolution increases, the initial dissipation rate decreases and significant dissipation does not occur until energy is transferred to sufficiently small scales. For the three highestresolution cases, there is a well-defined time of maximum energy dissipation rate at around $T=9.2$. For these cases, the peak dissipation rate $\epsilon_{T}$ appears to decrease slowly with increasing resolution, and hence with $R e$. However, the possibility of a non-zero limiting value as $R e \rightarrow \infty$ cannot be ruled out (note the plateau after the peak). This, together with the apparent insensitivity of $T$ to $R e$, is consistent with Kolmogorov's spirit and does not preclude the possibility of a finite-time singularity in the inviscid dynamics. The slow decrease of $\epsilon_{T}$, but without a tendency to vanish as $R e \rightarrow \infty$, is in apparent contradiction with the earlier numerical calculations of 


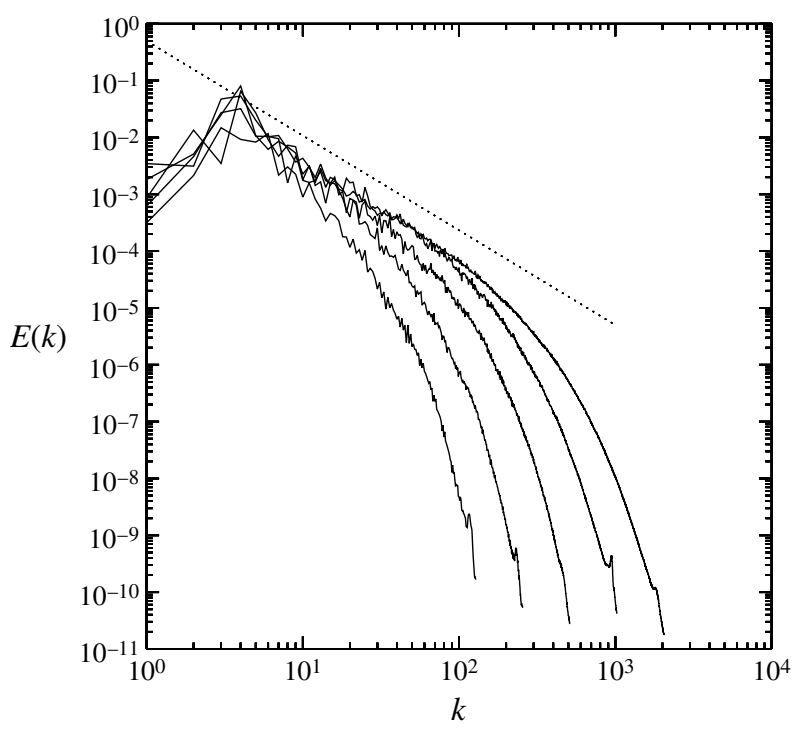

FIGURE 3. The energy spectra $E(k)$ versus $k$ for the same series of integrations with resolutions as in figure 2 . The reference line has a slope of $-5 / 3$.

Ohkitani \& Yamada (1997), which suggested a power-law decay of $\epsilon_{T}$ with $R e$. This could be due simply to the lack of resolution in the earlier work. Another possible explanation is that the initial condition in Ohkitani \& Yamada (1997) was one in which almost all energy was concentrated at the domain scale, which necessarily results in a restricted direct energy cascade, as discussed in the preceding section.

Figure 3 shows the energy spectrum $E(k)$ versus $k$ for the same series of simulations at $t=20$, by which time the direct cascade is well established (spectra at $t=T \approx 9.2$ are slightly steeper and not shown). Spectral slopes are steeper than $k^{-5 / 3}$ at low $R e$ but become shallower towards this spectrum as $R e$ increases. In the case of the highest $R e$, a clean $k^{-5 / 3}$ inertial range is obtained across more than a decade of wavenumbers. Note in particular that the degree of the small kinks at the high-wavenumber tails is about the same in each case, confirming our choice of scaling for $v$ with $k_{\max }$ as described above. In passing, it is worth mentioning that insufficient viscosity (which would lead to more conspicuous kinks at the tails) is likely to yield inaccurate values of $\epsilon(t)$ and hence $\epsilon_{T}$. We emphasize, therefore, that it is essential to avoid underdissipating in numerical integrations if a weak dependence of $\epsilon(t)$ and $\epsilon_{T}$ on $R e$ is to be detected.

Next, we present and discuss the results from a second set of simulations, where the initial temperature field $\theta_{0}$ consists of Fourier modes $(7,7)$ and $(7,6)$, each with unit energy. This initial field is morphologically similar to the previous one and to the one used by Ohkitani \& Yamada (1997) and Constantin et al. (1998), having well-defined curves of large temperature gradient. A difference in the dynamics is that, for the present choice of $\theta_{0}$, only modes $(7 n, m)$, for integers $n$ and $m$, can be subsequently excited. However, the dual transfer is not hindered or suppressed in any way. We ran three simulations using the two highest resolutions with $v \approx 2 \times 10^{-5}, 4 \times 10^{-5}$ and $8 \times 10^{-5}$, which are significantly greater than those in the previous simulations at the same resolutions. The reason is that the present initial field has much more energy and is located at relatively smaller scales, hence requiring more viscous damping. 


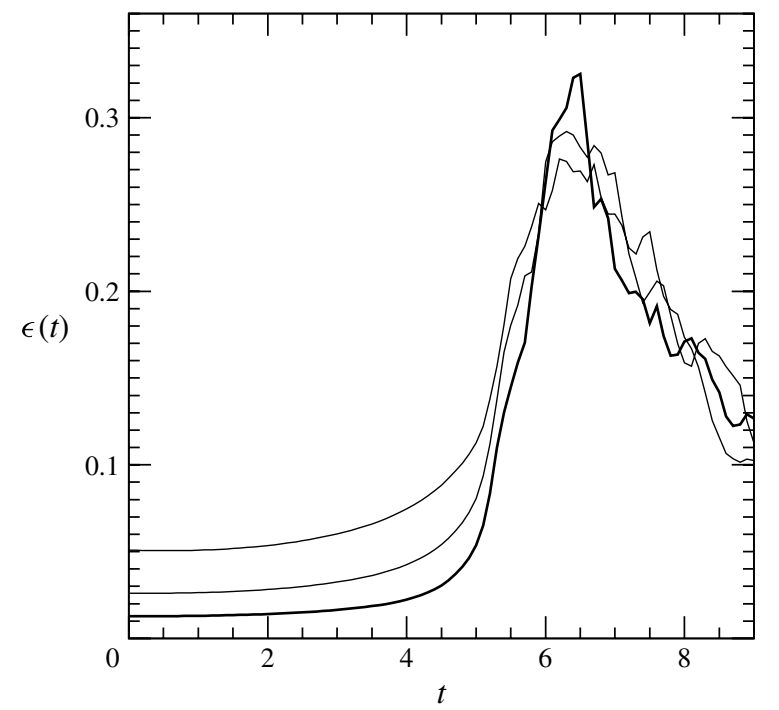

FIgURE 4. The energy dissipation rate $\epsilon(t)$ versus $t$ for numerical integrations with resolutions 2048 and 4096 . The diffusion coefficients are $v \approx 8 \times 10^{-5}$ for the lower resolution and $v \approx 4 \times 10^{-5}$ and $2 \times 10^{-5}$ for the higher resolution. Smaller $v$ corresponds to smaller $\epsilon_{0}$ and greater $\epsilon_{T}$.

Note that, owing to the more energetic initial temperature field (resulting in greater dissipation), the present Reynolds number for $v \approx 2 \times 10^{-5}$ is approximately the same as that of the preceding case for $v \approx 6.01 \times 10^{-6}$. Note further that, for these two cases, the ratios $\epsilon_{T} / \epsilon_{0}$, where $\epsilon_{0}=\epsilon(0)$, are approximately 26 and 18 , respectively. Since it is desirable for our investigation to have the ratio $\epsilon_{T} / \epsilon_{0}$ as high as possible, the present set of simulations, though not at higher $R e$, may better address the issue at hand.

Figure 4 shows the evolution of $\epsilon(t)$ from $t=0$ to $t=9$ for the simulations described above. Similar to figure $2, \epsilon(t)$ peaks roughly at the same time, around $t=6.5$, for all three Reynolds numbers under consideration. Unlike the previous case, for which $\epsilon_{T}$ appears to decrease slowly towards a non-zero value as $R e$ is increased, $\epsilon_{T}$ of the present case increases with $R e$, presumably growing towards a finite limit. We have no straightforward explanation for this difference, which seems immaterial as far as the possibility of non-zero limiting $\epsilon_{T}$ is concerned. However, one may argue that figure 4 better supports this possibility than figure 2 . Similar to the previous set of simulations, the energy spectrum (not shown) becomes shallower towards the classical spectrum as $R e$ increases.

Finally, it must be conceded that the range of Reynolds numbers considered here is insufficient to determine with any confidence whether the quantities $T$ and $\epsilon_{T}$ tend to some finite values, or perhaps exhibit a weak logarithmic dependence. Further investigation with significantly higher resolution would be necessary to address this issue convincingly. In any case, the theoretical analysis presented in $\S \S 2$ and 3 is independent of whether or not $T<\infty$ in the inviscid limit, and indeed remains valid even if $\epsilon_{T}$ tends to zero logarithmically with increasing $R e$.

\section{Conclusion}

We have studied SQG turbulence, showing evidence for the $k^{-5 / 3}$ energy spectrum in the inertial range, which has been predicted on the basis of Kolmogorov's 
phenomenology. This has been achieved by both analytical and numerical methods. In the former approach, we have derived the estimate $N \leqslant R e^{3 / 2}$ for the system's number of degrees of freedom $N$, where $R e$ is the Reynolds number defined in terms of the energy dissipation rate, the viscosity and the system's size. Upon approximating $N$ with the number of dynamically active Fourier modes, i.e. the modes within the energy inertial range, we deduce the result $\alpha=5 / 3$ for the exponent $\alpha$ of the power-law spectra $k^{-\alpha}$. This approximation also renders the scaling $R e^{1 / 2}$ for the exponential dissipation rate $r=v k_{v}^{2}$ at the dissipation wavenumber $k_{v}$. Given that $r$ is linear in $R e$ for Burgers flows, whose nonlinearity is fully quadratic, the sublinear scaling of $r$ with $R e$ in the present case (and in three-dimensional turbulence) is a manifestation of partial depletion of nonlinearity. In the latter approach, we have simulated the unforced SQG equation (1.1) up to a moderate resolution of $4096 \times 4096$ grid points. The results obtained include a series of spectra that become shallower for increasingly higher Reynolds numbers, appearing to tend to the predicted $k^{-5 / 3}$ spectrum. Furthermore, within the narrow range of accessible Reynolds numbers and for our choice of initial temperature field with relatively well-defined lines of large gradient, the energy dissipation rate appears to peak sharply in time and to be approximately independent of the Reynolds number. This behaviour is consistent with a positive limiting dissipation rate in the inviscid limit.

Although the dynamical parameter $\epsilon_{T}$ plays an important role in the present study, its detailed behaviour does not affect our results or conclusion in a significant way. In the event that $\epsilon_{T}$ vanishes logarithmically, the Reynolds number $R e$ would have a logarithmic correction to its conventional form $\propto 1 / \nu$ and the key result $N \leqslant R e^{3 / 2}$ remains intact. In addition, the arguments leading to the conclusion $\alpha=5 / 3$ remain valid, although it would not be possible to express the energy spectrum in the classical form $C_{K} \epsilon_{T}^{2 / 3} k^{-5 / 3}$ without modifications. This situation is analogous to two-dimensional turbulence, for which the -1 slope of the predicted enstrophy spectrum $Z(k) \propto \eta^{2 / 3} k^{-1}$ is not invalidated by the fact that the enstrophy dissipation rate $\eta$ slowly vanishes in the inviscid limit for such a slope (Tran \& Dritschel 2006). However, the vanishing factor $\eta^{2 / 3}$ may not give the correct collapsing rate of $Z(k)$ when a finite vorticity reservoir forever spreads out over an increasingly wider inertial range. Indeed, for such a vorticity redistribution, Dritschel et al. (2007) show that $Z(k)$ scales as $(\ln R e)^{-1} k^{-1}$, thereby requiring $\eta$ in the above expression of $Z(k)$ to behave as $(\ln R e)^{-3 / 2}$. This vanishing rate for $\eta$ is relatively more rapid than the recent mathematical estimate $(\ln R e)^{-1 / 2}$ of Tran \& Dritschel (2006) and numerical estimate $(\ln R e)^{-1}$ of Dritschel et al. (2007).

The behaviour of the maximum energy dissipation rate $\epsilon_{T}$ and the corresponding time $T$ is an interesting problem, not only for the present case but also for other fluid equations, whose solutions are known to be regular in the presence of viscosity but not known to be so otherwise. By monitoring $\epsilon_{T}$ and $T$ against $\nu$, one can obtain invaluable insights into the question of inviscid singularities. Indisputable evidence for a positive answer to this question would be that both $\epsilon_{T}$ and $T$ become independent of $v$ for small $v$. In this case, the smooth viscous solution presumably approaches the (weak) inviscid solution. Note that, in general, $T$ is not related to the inviscid singular time, which would correspond to the onset of sharp increase in $\epsilon(t)$ as in the case of Burgers flows (Tran \& Dritschel 2010). In the event that $T$ grows without bound as $v$ is decreased (irrespective of the behaviour of $\epsilon_{T}$ ), then finite-time inviscid singularities are highly unlikely. Since viscous dynamics are much easier to simulate than their inviscid counterparts, the above approach can be particularly useful in addressing the issue of inviscid singularities. 
We thank the referees for constructive comments, which helped to improve the presentation of this paper. L.A.K.B. was supported by an EPSRC post-graduate studentship.

\section{REFERENCES}

Capet, X., Hua, B. L., Lapeyre, G. \& McWilliams, J. C. 2008 Surface kinetic energy transfer in surface quasi-geostrophic flows. J. Fluid Mech. 604, 165-174.

Constantin, P., E, W. \& Titi, E. S. 1994 Onsager's conjecture on the energy conservation for solutions of Euler's equation. Commun. Math. Phys. 165, 207-209.

Constantin, P., Foias, C. \& TemaM, R. 1988 On the dimension of attractor in two-dimensional turbulence. Physica D 30, 284-296.

Constantin, P., MAJdA, A. \& TABAK, E. 1994 Formation of strong fronts in the 2D quasi-geostrophic thermal active scalar. Nonlinearity 7, 1495-1533.

Constantin, P., Nie, Q. \& SChÖRGHOFER, N. 1998 Nonsingular surface quasi-geostrophic flow. Phys. Lett. A 241, 168-172.

Doering, C. R. \& GibBon, J. D. 1991 Note on the Constantin-Foias-Temam attractor dimension estimate for 2-dimensional turbulence. Physica D 48, 471-480.

Dritschel, D. G., Tran, C. V. \& ScotT, R. K. 2007 Revisiting Batchelor's theory of two-dimensional turbulence. J. Fluid Mech. 591, 379-391.

Held, I. M., Pierrehumbert, R. T., Garner, S. T. \& Swanson, K. L. 1995 Surface quasi-geostrophic dynamics. J. Fluid Mech. 282, 1-20.

Hou, T. Y. \& LI, R. 2006 Dynamic depletion of vortex stretching and non-blowup of the 3D incompressible Euler equations. J. Nonlinear Sci. 16, 639-664.

Hou, T. Y. \& LI, R. 2008 Blowup or no blowup? The interplay between theory and numerics. Physica D 237, 1937-1944.

JuCKeS, M. N. 1994 Quasi-geostrophic dynamics of the tropopause. J. Atmos. Sci. 51, 2756-2768.

Kiselev, A., Nazarov, F. \& Volberg, A. 2007 Global well-posedness for the critical 2D dissipative quasi-geostrophic equation. Invent. Math. 167, 445-453.

OHKITANI, K. 2011 Growth rates analysis of scalar gradient in generalized surface quasi-geostrophic equations of ideal fluids. Phys. Rev. E 83, 036317.

OHKITANi, K. \& YAMADA, M. 1997 Inviscid and inviscid-limit behaviour of a surface quasigeostrophic flow. Phys. Fluids 9, 876-882.

Onsager, L. 1949 Statistical hydrodynamics. Nuovo Cimento (Suppl.) 6, 279-287.

Pedlosky, J. 1987 Geophysical Fluid Dynamics, 2nd edn. Springer.

Pierrehumbert, R. T., Held, I. M. \& Swanson, K. L. 1994 Spectra of local and nonlocal two-dimensional turbulence. Chaos, Solitons Fractals 4, 1111-1116.

Robinson, J. C. 2003 Low dimensional attractors arise from forcing at small scales. Physica D 181, 39-44.

ScotT, R. K. 2006 Local and nonlocal advection of a passive scalar. Phys. Fluids 18, 116601.

Smith, K. S., Boccaletti, G., Henning, C. C., Marinov, I., Tam, C. Y., Held, I. M. \& VAllis, G. K. 2002 Turbulent diffusion in the geostrophic inverse cascade. J. Fluid Mech. 469, $13-48$.

Temam, R. 1997 Infinite-Dimensional Dynamical Systems in Mechanics and Physics, 2nd edn. Springer.

TRAN, C. V. 2009 The number of degrees of freedom of three-dimensional Navier-Stokes turbulence. Phys. Fluids 21, 125103.

Tran, C. V. \& Blackbourn, L. 2009 Number of degrees of freedom of two-dimensional turbulence. Phys. Rev. E 79, 056308.

Tran, C. V. \& Dritschel, D. G. 2006 Vanishing enstrophy dissipation in two-dimensional Navier-Stokes turbulence in the inviscid limit. J. Fluid Mech. 559, 107-116.

Tran, C. V. \& Dritschel, G. D. 2010 Energy dissipation and resolution of steep gradients in one-dimensional Burgers flows. Phys. Fluids 22, 037102.

Tran, C. V., Dritschel, D. G. \& ScotT, R. K. 2010 Effective degrees of nonlinearity in a family of generalized models of two-dimensional turbulence. Phys. Rev. E 81, 016301. 